\title{
Verteilte Kommunikations- und Sensornetzwerke für die kooperative, intelligente Kreuzung von Morgen
}

Andreas Herrmann, Sebastian Naumann

Institut für Automation und Kommunikation e. V. Magdeburg, Bereich Verkehrstelematik, WernerHeisenberg-Str. 1, 39106 Magdeburg

Tel.-Nr., E-Mail-Adresse(n): +49 (391) 9901440 / +49 (391) 9901 443, andreas.herrmann@ifak.eu / sebastian.naumann@ifak.eu

\section{Problemstellung}

Die Sicherung der Mobilität ist nach wie vor eine der Voraussetzungen für das Funktionieren unseres Wirtschaft- und Gesellschaftssystems. Die ökonomischen Herausforderungen der Zukunft, die Begrenzung der zur Verfügung stehenden Rohstoffe und Ressourcen und der bereits spürbare demografische Wandel erfordern neues, teils unkonventionelles Denken, innovative Konzepte und nachhaltige Mobilitätsstrategien. Soll die Mobilität auch in Zukunft erhalten werden, ist neben der effizienten Nutzung der vorhandenen Ressourcen der Erhalt einer leistungsfähigen Verkehrsinfrastruktur von entscheidender Bedeutung.

Gerade in urbanen Räumen ist zu beobachten, dass der Verkehr in den vergangenen Jahren deutlich an Komplexität gewonnen hat. Die resultierenden Überlastungen der Verkehrsnetze - durch Übernachfrage der Verkehrswege in Spitzenstunden, durch Baustellen oder durch singuläre Störungen - können zukünftig nur noch eingeschränkt durch Neu- bzw. Ausbau der Verkehrsinfrastrukturen vermieden werden. Zur Lösung dieses Zielkonfliktes sind unter Berücksichtigung von wirtschaftlicher Effizienz sowie gesellschafts- und umweltpolitischen Ansprüchen neue Ansätze im Bereich der Verkehrstelematik und ihrer zunehmend kooperativ organisierten Systeme gefragt. Eine zukunftsweisende Möglichkeit zur effizienten Lösung aktueller und künftiger verkehrlicher Herausforderungen wird besonders in der Nutzung kooperativer, intelligenter Verkehrssysteme im Mobilitätsmanagement gesehen.

\section{Intelligente und kooperative Verkehrssysteme}

\section{Einordnung in den Kontext}

Nach Definition der Europäischen Union sind Intelligente Verkehrssysteme (IVS) "hochentwickelte Anwendungen, die - ohne Intelligenz an sich zu beinhalten - darauf abzielen, innovative Dienste im Bereich verschiedener Verkehrsträger und des Verkehrsmanagements anzubieten, und die verschiedenen Nutzer mit umfassenderen Informationen zu versorgen und sie in die Lage zu versetzen, die Verkehrsnetze auf sicherere, koordiniertere und "klügere" Weise zu nutzen. Intelligente Verkehrssysteme kombinieren Telekommunikation, Elektronik und Informationstechnologie mit Verkehrstechnik zu dem Zweck, Verkehrssysteme zu planen, zu konzipieren, zu betreiben, zu warten und zu steuern.,

Kooperative Systeme stellen eine Teilgruppe der Intelligenten Verkehrssysteme dar, die untereinander kommunizieren und Informationen und Daten austauschen, um den Verkehr für den Verkehrsteilnehmer und sein Umfeld über das Maß hinaus sicherer, effizienter und umweltverträglicher zu machen, das durch nicht kooperative Intelligente Verkehrssysteme erreichbar ist. Es soll ein auf offenen

Kommunikationsstandards basierender Informationsaustausch zwischen den Akteuren erfolgen, der eine gemeinsame, hochaktuelle Wissensbasis schafft, welche von zukünftigen Fahrerinformations- und Assistenzsystemen interpretiert und genutzt werden kann.

Ein wesentlicher Aspekt bei der Interaktion und Kooperation verschiedener, unterschiedlich komplexer Verkehrssysteme ist, dass neben Fahrzeugen und Infrastruktur auch Betreiber, Fahrer und andere Straßenbenutzer kooperieren, um einen möglichst effizienten, sicheren, umweltgerechten und komfortablen Verkehrsablauf zu ermöglichen. Dabei gehen die Möglichkeiten kooperativer Systeme zur Erreichung dieser Zielsetzung deutlich über die einzelner, isolierter Systeme hinaus.

In diesem Kontext können kooperative, intelligente Verkehrssysteme als Katalysator sowohl für die Entwicklung neuer Anwendungen, Dienste und Technologien als auch für die erfolgreiche Einführung neuer Mobilitäts- und Verkehrskonzepte wie bspw. die Elektromobilität wirken. 


\section{Nationale und europäische Rahmenbedingungen}

Die Schaffung und Erhaltung einer effizienten, sicheren und umweltverträglicheren Mobilität im Personenund vor allem im Wirtschaftsverkehr ist eine Aufgabe, die nicht mehr allein durch einzelne

Nationalstaaten oder Bundesländer bewältigt werden kann. Daher hat die EU-Kommission im Jahr 2008 einen Aktionsplan zur Einführung Intelligenter Verkehrssysteme (ITS-Actionplan, Intelligent Transport Systems) und in 2010 die Richtlinie 2010/40/EU des Europäischen Parlaments und des Rates verabschiedet. Der ITS-Aktionsplan der EU dient einerseits der Strategiebestimmung und führt andererseits folgende allgemeine Ziele für eine effiziente, sichere und umweltverträglichere Mobilität auf [1]:

1. Nahtloses Verkehrsmanagement

2. Staubekämpfung im Fernverkehr und in den Ballungszentren

3. Wechselseitige Ergänzung der Verkehrsträger bei IST-Anwendungen und -Diensten

4. Verlässlichkeit im Wirtschaftsverkehr

5. Verbesserte Sicherheit von Straßen- und Verkehrsteilnehmern

6. Nahtlose Verkehrs- und Reisendeninformation in Echtzeit

7. Effizienter logistischer Betrieb

Aus den allgemeinen Zielen leiten sich spezifische Ziele ab. Der Fokus liegt hier insbesondere auf der Interoperabilität der Systeme und Komponenten durch Standardisierung, auf der Förderung und Sicherstellung des offenen ITS-Marktes in Europa, auf der Einrichtung von wirksamen, transparenten Abstimmungs- und Kooperationsprozessen aber auch auf dem Schutz der Privatsphäre. Die zeitgleich vorgelegte und in 2010 verabschiedete EU-Richtlinie 2010/40/EU sieht einen Rechtsrahmen für die umfassende Einführung und Nutzung intelligenter Verkehrssysteme in den einzelnen Mitgliedstaaten vor und dient der europaweiten Koordinierung. Bei der Ausgestaltung und Anwendung von Spezifikationen und Normen sowie der Umsetzung von Maßnahmen gibt die EU-Richtlinie die folgenden vorrangigen Bereiche vor [2]:

I. Optimale Nutzung von Straßen-, Verkehrs- und Reisedaten,

II. Kontinuität der IVS-Dienste in den Bereichen Verkehrs- und Frachtmanagement,

III. IVS-Anwendungen für die Straßenverkehrssicherheit und

IV. Verbindung von Fahrzeug und Verkehrsinfrastruktur.

Gemeinsames Ziel aller Beteiligten in EU, Bund und Ländern ist eine nachhaltige Verkehrspolitik, die den Personen- und Güterverkehr als Motor für Wirtschaft und Beschäftigung voranbringt, ohne die ökologischen Aspekte zu vernachlässigen, die sich nicht zuletzt aus der weitgehend gemeinsamen Nutzung der vorhandenen Verkehrswege und Ressourcen ergeben.

\section{Verteilte Sensornetzwerke zur Verkehrdatenerfassung im Kreuzungsbereich}

\section{Verfahren zur stationären und mobilen Verkehrsdatenerfassung}

Für die intelligente Steuerung von Lichtsignalanlagen an komplexen Kreuzungen ist die zuverlässige und möglicht vollständige Datenerfassung vor allem an den Haltelinien und an den Zufahrtsstrecken zwischen den Verkehrsknoten unabdingbar. Die gängigste Methode zur Verkehrsdatenerfassung beruht immer noch auf dem Einsatz ortsfester Detektoren wie in die Fahrbahn eingelassene Induktionsschleifen, die eigentlich primär der verkehrsabhängigen Steuerung eines signalisierten Knotenpunktes dienen, und an Brücken oder Masten angebrachte Passivinfrarotdetektoren. Alle wesentlichen Verkehrskenngrößen ergeben sich dabei aus der kontinuierlichen Zählung der Fahrzeuge und ggf. der Ermittlung der Fahrgeschwindigkeiten an den Messquerschnitten. Während Bundesautobahnen gegenwärtig am besten mit entsprechenden Messeinrichtungen ausgestattet sind, müssen im innerstädtischen Bereich die noch die lückenhaften Messdaten von Induktionsschleifen mit Hilfe von mathematischen Modellen vervollständigt werden. Deren Einsatz in der Praxis ist bisher allerdings eher die Ausnahme. Zudem weisen Forschungsarbeiten der jüngeren Vergangenheit auf die fehlende dauerhafte Zuverlässigkeit von Induktionsschleifen hin [8].

Weitere Möglichkeiten zur Erfassung von Verkehrsdaten sind die so genannten Floating Cars und neuerdings auch Floating Phones. Bei den Floating Cars handelt es sich um speziell ausgerüstete Fahrzeuge, die in regelmäßigen zeitlichen Abständen ihre momentane Position sowie ggf. weitere Daten an eine Zentrale melden. Die Stauerkennung funktioniert auf diese Weise gut, allerdings liefert ein im Stau stehendes Fahrzeug keine darüber hinaus gehenden Informationen insbesondere zur Staulänge und zu den Staugrenzen. Für den Einsatz als Floating Cars beliebt sind Taxis, da diese gegenüber den 
Fahrzeuge, die in regelmäßigen zeitlichen Abständen ihre momentane Position sowie ggf. weitere Daten an eine Zentrale melden. Die Stauerkennung funktioniert auf diese Weise gut, allerdings liefert ein im Stau stehendes Fahrzeug keine darüber hinaus gehenden Informationen insbesondere zur Staulänge und zu den Staugrenzen. Für den Einsatz als Floating Cars beliebt sind Taxis, da diese gegenüber den meisten anderen Fahrzeugen eine hohe Fahrleistung aufweisen. Zudem gibt es in Ballungsräumen üblicherweise eine große Anzahl an Taxis. Des Weiteren ist die fahrzeugseitig erforderliche Technik zur Fahrzeugortung und Datenkommunikation in der Regel bereits vorhanden.

Bei den Floating Phones werden Verkehrsdaten mit Hilfe von Mobiltelefonen gewonnen. Grundlage ist dabei die Ortung von Mobiltelefonen anhand der jeweiligen Funkzelle und der jeweiligen Signalstärke, wobei durch eine Funkzelle bzw. einen Sendemast jeweils ein bestimmtes räumliches Gebiet abgedeckt wird. Rückschlüsse auf die Verkehrslage können dann durch die Registrierung der Zellwechsel, d. h. ein Mobiltelefon verlässt eine Funkzelle und tritt in eine andere Funkzelle ein, und deren Zeitpunkte gezogen werden. Auf diese Weise ist zwar eine groß- allerdings keine kleinräumige Verkehrsdatenerfassung und Stauerkennung möglich.

\section{Mobile Erfassung von Verkehrsdaten durch Beobachtung des Gegenverkehrs}

Zur Verbesserung des gegenwärtig noch unbefriedigenden Zustands wurde vom Institut für Automation und Kommunikation e. V. Magdeburg die Idee entwickelt, mit Hilfe eines speziellen Sensors von einem fahrenden Fahrzeug aus den Gegenverkehr zu beobachten. In Anlehnung an die Floating Cars, die im Verkehr mitschwimmen und aufgrund der eigenen Position und Fahrgeschwindigkeit sowie ggf. weiterer Sensordaten Verkehrslageinformationen generieren - die Floating Car Data (FCD) -, wird das Prinzip der Gegenverkehrsbeobachtung als Floating Car Observer (FCO) bezeichnet. Es ist in Abbildung 1 vereinfacht dargestellt.

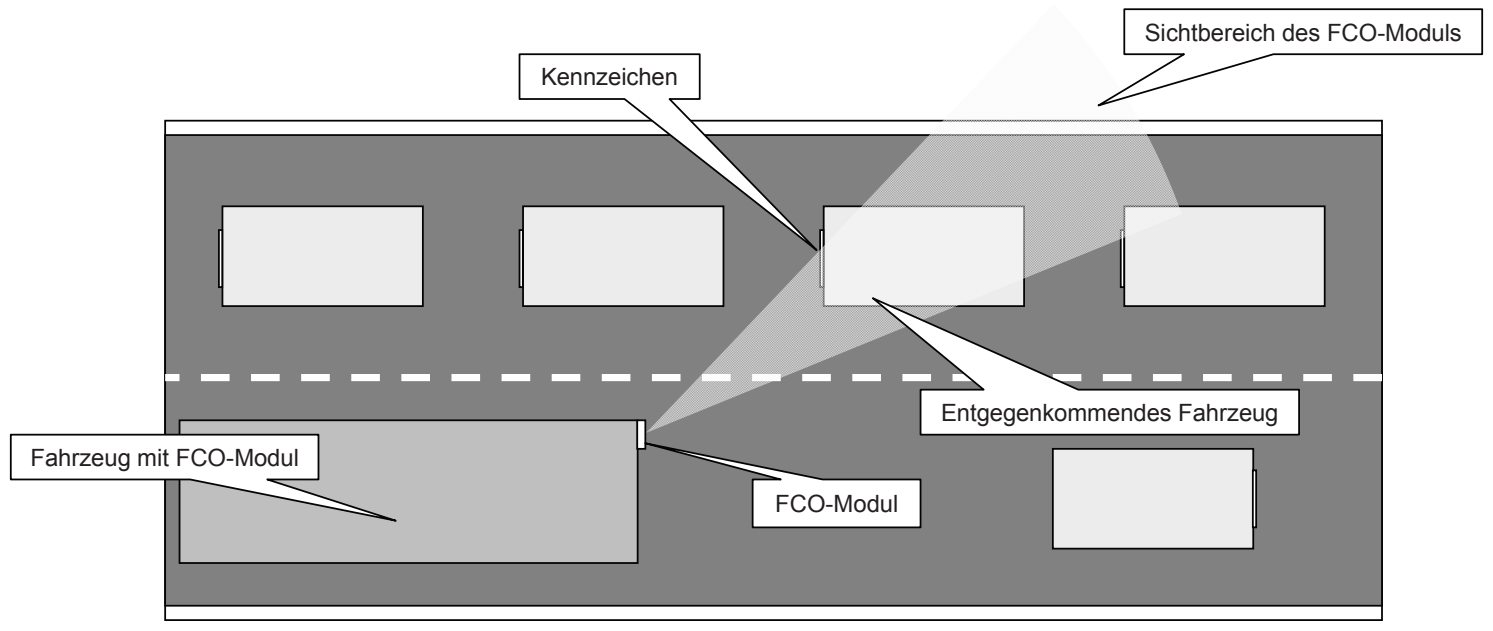

Abbildung 1: Schematisches Funktionsprinzip des Floating Car Observers [4]

Das entwickelte FCO-Modul besteht im Wesentlichen aus einem Infrarotstrahler, einem CMOSKamerasensor und einem Mikrocontroller. Der Infrarotstrahler besteht aus mehreren Infrarot-LED und dient insbesondere dazu, die Kennzeichen der entgegenkommenden Fahrzeuge, welche mit einer retroreflektierenden Schicht versehen sind, zu beleuchten und dadurch im aufgenommenen Kamerabild vom Hintergrund abzuheben. Bei der Suche nach einem Fahrzeugkennzeichen im Kamerabild wird keine optische Zeichenerkennung (OCR) durchgeführt. Die Kennzeichenerkennung beruht vielmehr auf der weitestgehend eindeutigen Form, die ein Kennzeichen innerhalb des Straßenraums aufweist. Anhand der Bewegung eines Kennzeichens durch den Bildbereich der Kamera in mehreren aufeinander folgenden Kamerabildern lässt sich schließlich die Geschwindigkeit des entsprechenden Fahrzeugs ermitteln. In Abbildung 2 ist der schematische Aufbau des FCO-Moduls dargestellt.

Für die Erkennung der Kennzeichen und der Geschwindigkeit eines entgegenkommenden Fahrzeugs sind die folgenden Schritte in der Bilddatenverarbeitung notwendig [4]:

- Objekterkennung

- Objektverfolgung

- Geschwindigkeitsermittlung

Die Schritte Objekterkennung und Objektverfolgung müssen für jedes der 60 pro Sekunde aufgenommenen Kamerabilder abgearbeitet werden. Die Geschwindigkeitsermittlung wird nur dann 
ausgeführt, wenn ein entgegenkommendes Fahrzeug den Erfassungsbereich der Kamera passiert hat (Abbildung 3).

Die Objekterkennung, d. h. die Identifizierung der evtl. vorhandenen Fahrzeugkennzeichen im Kamerabild, ist der rechenintensivste Schritt. Aufgrund der Bildaufnahmerate von 60 Bildern/s steht für die Objekterkennung und die Objektverfolgung auf der FCO-Hardware eine maximale Rechenzeit von max. 16,6 ms pro Bild zu Verfügung.

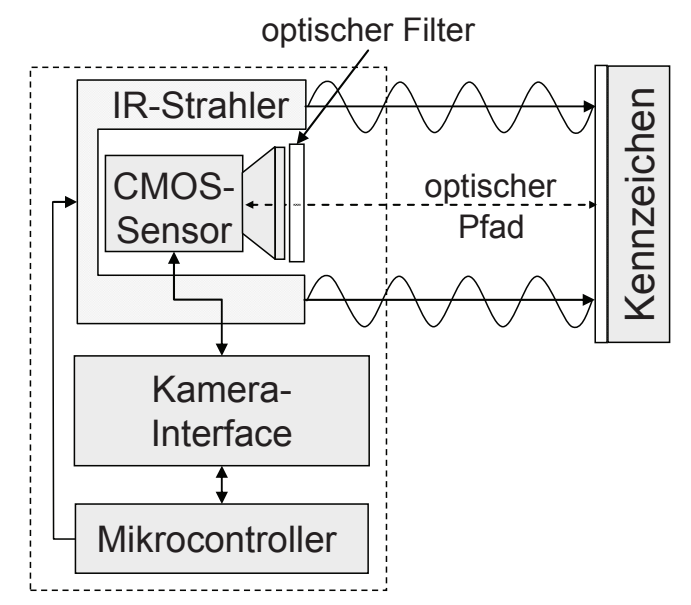

Abbildung 2: Schematischer Aufbau des FCO-Moduls

Zunächst wird das Kamerabild binarisiert, d. h. alle Pixel, deren Helligkeitswert unterhalb einer bestimmten Schwelle liegt, erhalten den Helligkeitswert 0 (schwarz) und alle anderen den Helligkeitswert 1 (weiß). Nach der Binarisierung müssen zusammenhängende helle Bereiche gefunden und anhand verschiedener Filterkriterien geprüft werden, ob es sich wirklich um ein Fahrzeugkennzeichen handelt. Grundsätzlich arbeitet der Algorithmus nach dem Prinzip, ein Kamerabild solange rekursiv horizontal und vertikal in zusammenhängende Bildbereiche zu zerteilen, bis nur noch unteilbare Bildbereiche übrig bleiben.

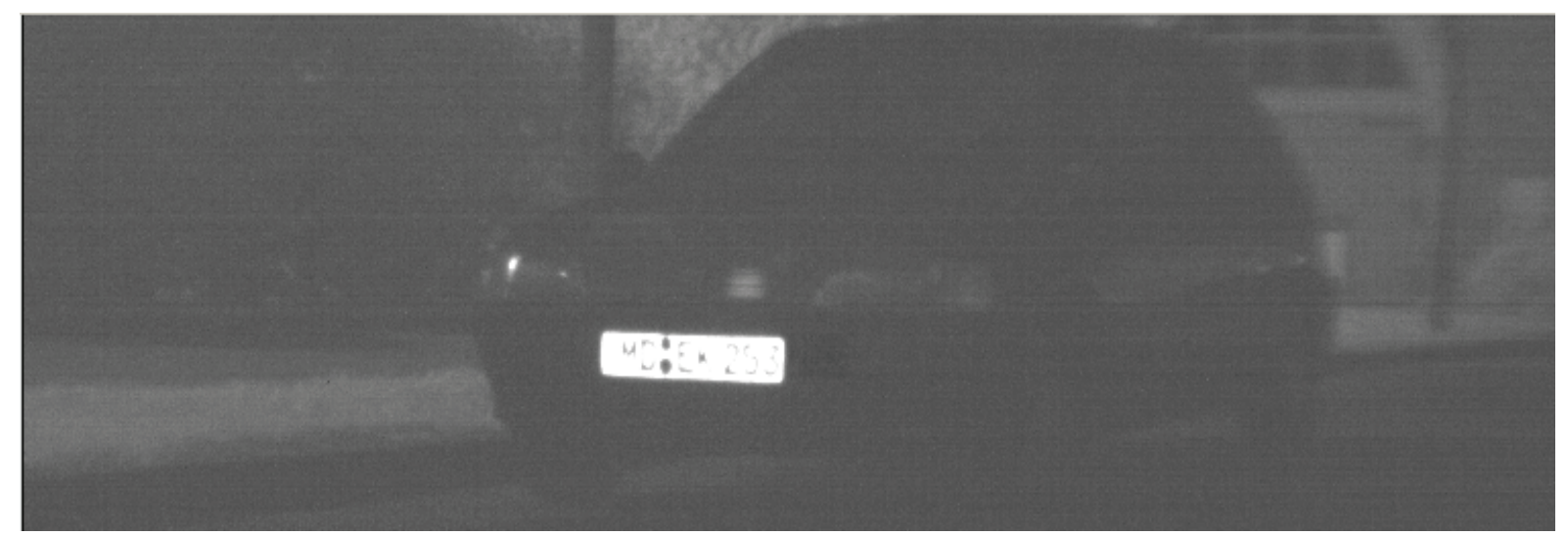

Abbildung 3: Originales FCO-Kamerabild

Die gefundenen Objekte, d. h. die übrig gebliebenen unteilbaren Bildbereiche, werden anschließend anhand der folgenden Kriterien gefiltert:

- $\quad$ minimale und maximale Breite

- minimale und maximale Höhe

- $\quad$ minimaler Füllgrad, wobei Füllgrad das Verhältnis der Anzahl von Bildpunkten mit dem Helligkeitswert 1 zur Gesamtzahl der Bildpunkte im betrachteten Bildbereich) meint

- minimales und maximales Seitenverhältnis

- maximaler Verkippungswinkel (Abweichung von der „normalen“ horizontalen Ausrichtung der Objekte bzw. Kennzeichen)

Die Objektverfolgung beschäftigt sich damit, ein Fahrzeugkennzeichen in aufeinander folgenden Bildern wieder zu erkennen. Dies erfolgt über die Vorhersage der Position eines Fahrzeugkennzeichens im 
nächsten Kamerabild aufgrund der vorherigen Positionen. Alle Positionen eines Kennzeichens im Bildbereich der Kamera werden zu einem Verlauf zusammengefasst.

Nach der Abarbeitung des Schrittes Objekterkennung ist der Objektverfolgung Folgendes gegeben:

- Menge von Kennzeichen, die im aktuellen Kamerabild identifiziert wurden

- Menge von bestehenden Verläufen, denen evtl. eines der Kennzeichen aus dem aktuellen Kamerabild zugeordnet werden kann

Zunächst wird nun eine Matrix erstellt, die eine Aussage über die Korrelation zwischen den Verläufen und den Kennzeichen trifft. Dazu wird mit Hilfe des Gauss-Newton-Verfahrens zur Polynominterpolation für jeden Verlauf ein quadratisches Polynom errechnet, welches die Kennzeichenbewegung durch den Bildbereich der Kamera abbildet. Das quadratische Polynom dient anschließend zur Berechnung der erwarteten Kennzeichenposition im nächsten Kamerabild. Obwohl die Kennzeichenbewegung durch eine Winkelfunktion beschrieben wird, wurde hier die quadratische Funktion verwendet, da praktische Versuche gezeigt haben, dass die quadratische Funktion für die Anwendungsfälle ausreichend genau ist und darüber hinaus einfacher und schneller berechnet werden.

Wird ein Verlauf im Zuge der Objektverfolgung beendet, so wird anschließend die Geschwindigkeit des zugehörigen Fahrzeugs berechnet. Grundsätzlich erfolgt die Berechnung der Begegnungsgeschwindigkeit zwischen dem FCO-Modul und dem entgegenkommendem Fahrzeug nach der Formel

$v=\frac{s}{t}$

wobei sich der Weg $s$ und die Zeit $t$ aus zwei ausgewählten Kamerabildern und den dort erkannten Kennzeichen ergeben. In Abbildung 4 ist das Prinzip skizziert.

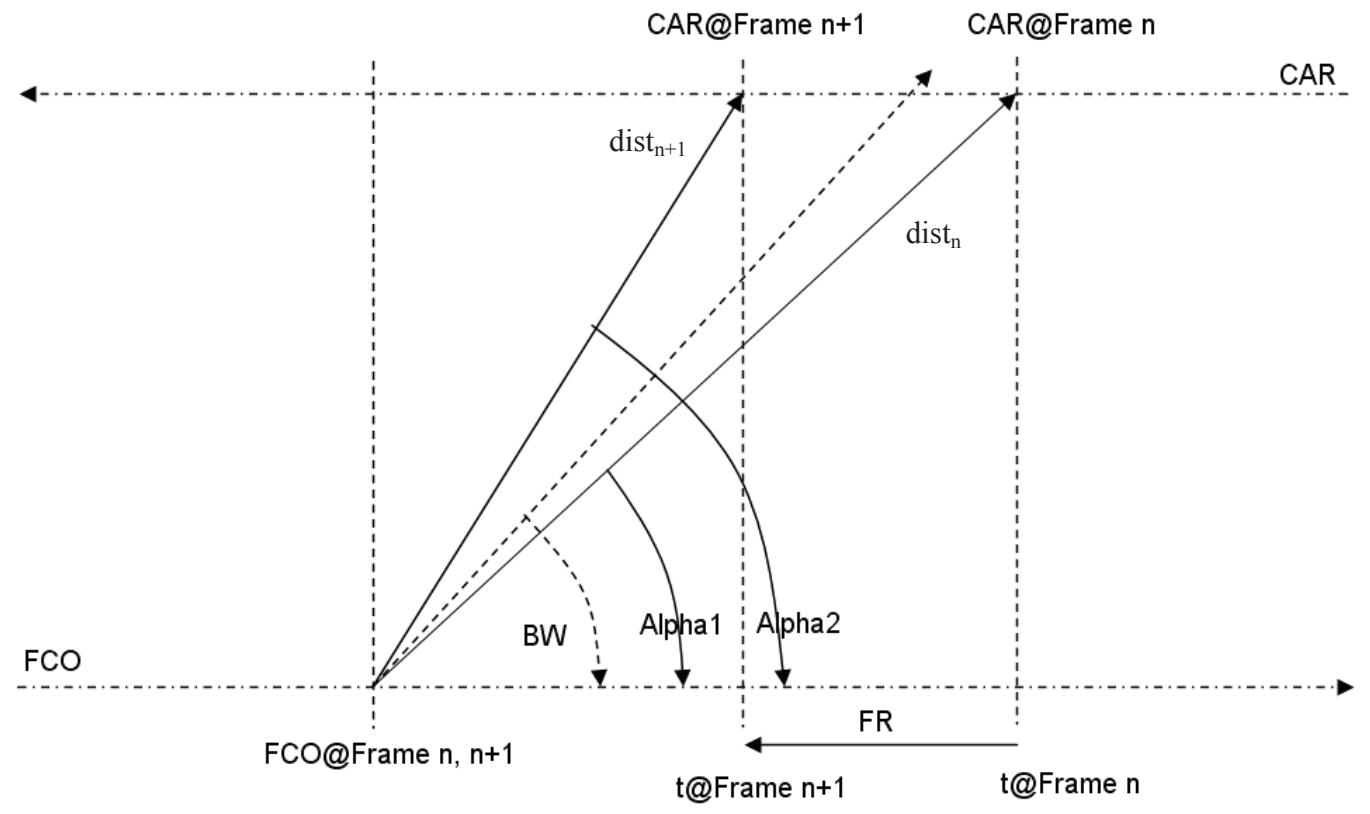

Abbildung 4: Geschwindigkeitsberechnung anhand zweier ausgewählter Kennzeichen eines Verlaufes Das implementierte Verfahren berücksichtigt alle Bilder eines Verlaufs, um mittels linearer Regressionsanalyse eine höhere Genauigkeit bei der Geschwindigkeitsermittlung zu erzielen. Besonders bei geringen Relativgeschwindigkeiten, wie sie bei Störungen oder Staus im Verkehr überwiegend zu erwarten sind, trägt die größere Anzahl an verwertbaren Bildern dazu bei, systembedingte Messfehler sowie Messausreißer herauszufiltern.

Die Zeit $t$ als Differenz

$t=t_{n+1}-t_{n}$

zwischen zwei aufeinanderfolgenden Kamerabildern ist bekannt, konstant und einzig von der Framerate des verwendeten Kamerasensors abhängig. Die Entfernung dist eines Kennzeichens zum FCO-Modul wird aus der Höhe des Kennzeichens im Kamerabild unter der Annahme, dass es sich um ein einzeiliges 
Kennzeichen mit einer realen Höhe von $110 \mathrm{~mm}$ handelt, gewonnen. Der Weg $s$ berechnet sich dann aus der Formel

$s=\cos \left(\alpha_{1}\right) \cdot$ dist $_{n}-\cos \left(\alpha_{2}\right) \cdot$ dist $_{n+1}$

Von der auf diese Weise errechneten Begegnungsgeschwindigkeit muss abschließend die Eigengeschwindigkeit des FCO abgezogen werden. Die benötigten Sichtwinkel Alpha1 und Alpha2 können aus den Koordinaten der jeweiligen Kennzeichenobjekte im Bild und unter Berücksichtigung des Blickwinkels BW, welcher dem Montagewinkel des FCO-Sensors am Fahrzeug entspricht, errechnet werden.

Durch die verwendeten Algorithmen konnte eine weitgehende Unabhängigkeit bezüglich der Montagehöhe und der Ausrichtung des FCO-Moduls erreicht werden. Weiterhin konnte so auch der seitliche Abstand der entgegenkommenden, zu erfassenden Fahrzeuge ermittelt werden, womit auch eine Zuordnung der Fahrzeuge und deren Geschwindigkeiten zu den entsprechenden Fahrspuren erfolgen kann.

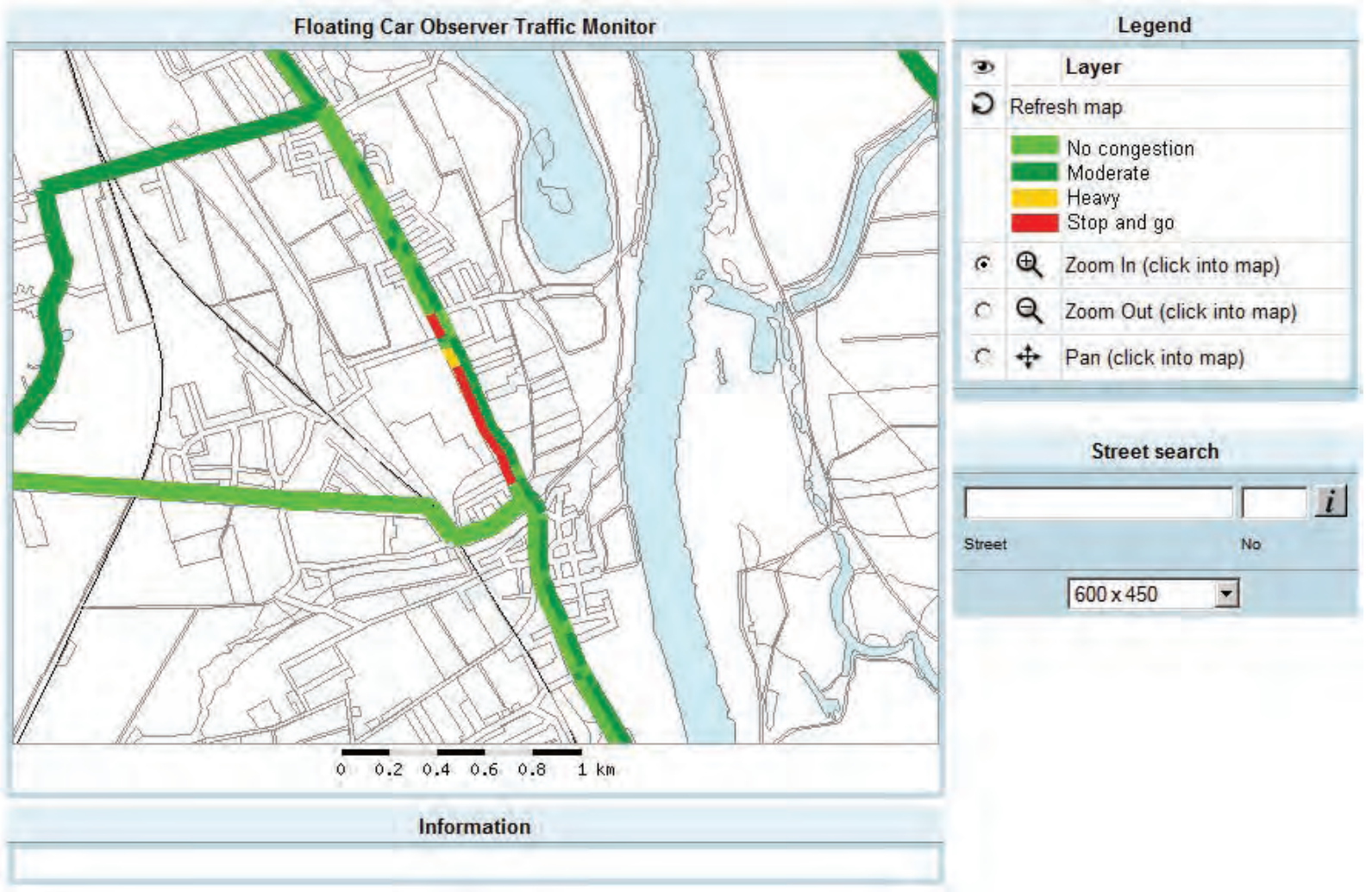

Abbildung 5: Grafische Darstellung der Verkehrslage anhand von FCO-Sensordaten [4]

Die vom FCO-Sensor erfassten Verkehrsdaten, also jedes einzelne erkannte Fahrzeug mit geografischer Position, Fahrtrichtung und gemessener Geschwindigkeit, werden über ein geeignetes Datennetzwerk (Mobilfunk, Automotive-WLAN) an eine Zentrale oder auch straßenseitige Kommunikationseinrichtungen (Road Site Stations) zur weiteren Verbreitung und Verarbeitung gesendet. Anhand der geografischen Position und der Fahrtrichtung können in einer digitalen Karte der entsprechende Straßenabschnitt sowie die genaue Position auf diesem bestimmt werden.

Zur Berechnung der Reisezeit auf einem Straßenabschnitt wird zunächst die Reisegeschwindigkeit ermittelt. Auf einem Abschnitt, für den Fahrzeugdaten vorliegen, wird aus allen gemeldeten Fahrzeuggeschwindigkeiten die Durchschnittsgeschwindigkeit berechnet. Für Abschnitte, für die keine Fahrzeugdaten vorliegen, entspricht die Reisegeschwindigkeit der dortigen zulässigen Höchstgeschwindigkeit. Die Reisezeit für einen Abschnitt ergibt sich dann aus der jeweiligen Reisegeschwindigkeit und Linkabschnittslänge. Die Reisezeit für einen Abschnitt entspricht der Summe der Reisezeiten aller Straßenabschnitte. Abbildung 5 zeigt beispielhaft die grafische Darstellung der unterschiedlichen durchschnittlichen Reisegeschwindigkeiten in einem vierstufigen Farbschema (frei fließender Verkehr, dichter Verkehr, Staugefahr, Stau). Die aufbereiteten Daten können mit anderen Daten fusioniert und in übergeordnete Systeme und Modelle zur Verkehrslageerfassung integriert oder auch direkt - beispielsweise zur Messung von Rückstaulängen an LSA-gesteuerten Kreuzungen verwendet werden [5]. 


\section{Monitoring von Lichtsignalanlagen an Kreuzungen}

Für das innerstädtische Verkehrsmanagement ist die Optimierung der Lichtsignalanlagen-Steuerung das wichtigste Werkzeug, mit dem die Verkehrsqualität für alle Verkehrsarten gesteuert werden kann. Eine eingehende Überprüfung der Steuerungsqualität von Lichtsignalanlagen (LSA) nach deren Inbetriebnahme und vor allem in der weiteren Betriebszeit birgt große Potenziale zur Effizienzsteigerung der Verkehrsabwicklung. Dies findet aber nicht zuletzt aus Kostengründen nur selten statt. Daher beschränkt sich die Überwachung von LSA größtenteils auf deren prinzipielle Funktionsfähigkeit.

Zur Ermittlung von Schaltzuständen der LSA-Signalgeber sind mehrere direkte und indirekte Verfahren bekannt. Die direkten Verfahren erfordern aber einen Eingriff in die Steuerungs- und Kontrolleinrichtung der Signalanlage bzw. einen Fernzugang über Kabel oder Funk. Hierbei können oft nur wenige Anlagen im Straßennetz simultan überwacht werden. Die indirekten Verfahren basieren im Wesentlichen auf einer Korrelation der Bewegungsmuster der die Lichtsignalanlage passierenden Fahrzeuge und dem aktuellen Schaltzustand der Signalgeber.

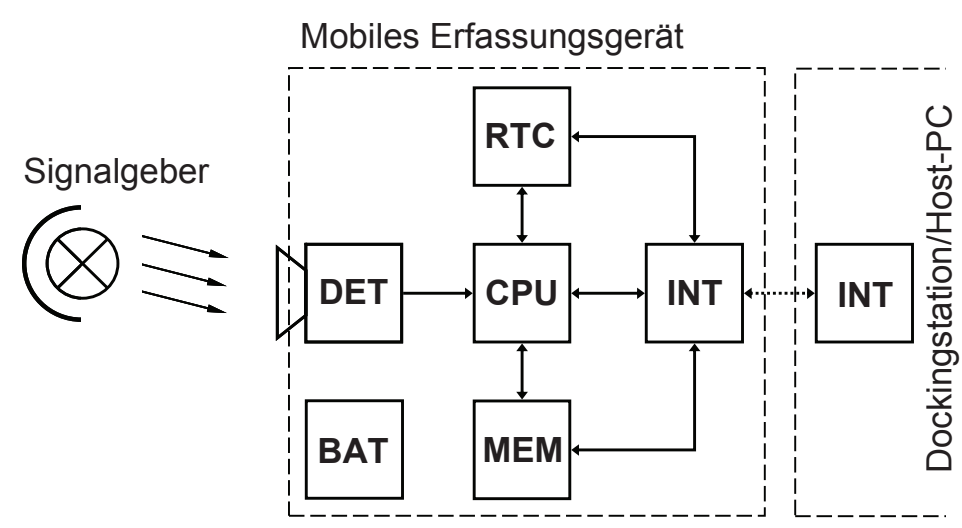

\section{Abbildung 6: Systemkonzept des Sensors zur verteilten Erfassung der LSA-Schaltzustände}

LSA-Monitoring ist die Beobachtung und Aufzeichnung wichtiger Anlagenparameter und -zustände während des Betriebs. Es dient der Bewertung der Steuerungsqualität der gesamten Anlage - auch im Zusammenspiel mit benachbarten Knoten und dem öffentlichen Verkehr (ÖV). Die Idee des nachfolgend beschriebenen, vom ifak entwickelten elektronischen Sensors (Abbildung 6) besteht darin, den Signalisierungsverlauf einer LSA über den Ein- und Ausschaltzeitpunkt der Signalgeber rückwirkungsfrei, d. h. ohne Eingriff in die Anlage zu ermitteln.

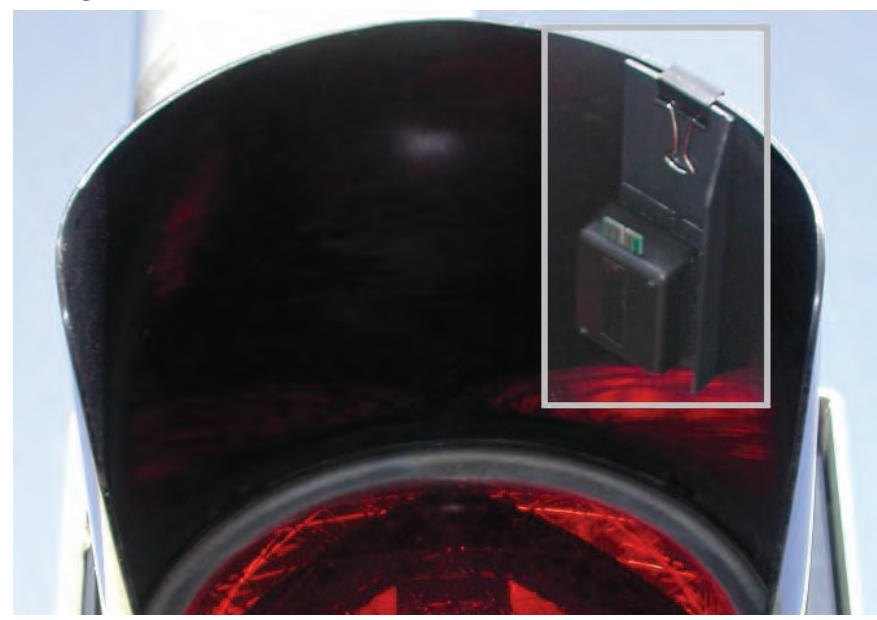

Abbildung 7: Montagepunkt des Sensors an einer Lichtsignalanlage

Das einfallende Licht eines Signalgebers wird in einer Detektionseinheit (DET) erfasst, charakteristische Änderungen der Signalamplitude erkannt und als elektrisches Signal an die zentrale Verarbeitungseinheit (CPU) weitergeleitet. Dort wird das Signal ausgewertet, mit einem aktuellen Zeitstempel aus dem Zeitgeber (RTC) versehen und in einem Pufferspeicher (MEM) abgelegt. Alle Komponenten des mobilen Erfassungsgerätes werden aus einer Batterie (BAT) gespeist. Über eine vordefinierte, im Zeitgeber (RTC) gespeicherte Ein- und Ausschaltzeit kann die Datenerfassung des mobilen Gerätes synchron zu baugleichen Geräten an anderen Signalgebern aktiviert und deaktiviert werden. 
Das ermöglicht die zeitsynchrone und großräumige Auswertung von Schaltvorgängen an verteilten Lichtsignalanlagen. Alle für den Betrieb wichtigen Parameter des Gerätes werden im Zeitgebermodul (RTC) gespeichert. Die Initialisierung und Parametrierung sowie das Auslesen der erfassten Daten erfolgen am PC. Über die geräteinterne Schnittstelle (INT) und das Gegenstück in der Dockingstation werden die entsprechenden Daten und Informationen ausgetauscht.

Während der mehrtägigen Datenerfassung arbeitet das Gerät autark und somit unabhängig von der an der Signalanlage vorhandenen technischen Infrastruktur. Durch die kompakte Bauweise und die Art der Montage wird eine Verdeckung der Signalgeber vermieden und somit eine Gefährdung des Straßenverkehrs verhindert (Abbildung 7).

Zur Erprobung der Erfassungsgeräte und zur Überprüfung des Systemansatzes wurden mehrere Pilotversuche u. a. in Magdeburg, Leipzig und Düsseldorf durchgeführt. In diesen praktischen Versuchen wurden bis zu 30 Erfassungsgeräte gleichzeitig an mehr als 10 Knotenpunkten eingesetzt. Die zuverlässig erfassten Daten wurden mit einer im ifak entwickelten Software aufbereitet und ausgewertet. Dadurch ist der Vergleich mit den verkehrstechnischen Unterlagen anhand von Signalzeitenplänen sowie Phasendarstellungen möglich. Bei der Analyse mehrerer Knotenpunkte kann außerdem auch die Koordinierungsgeschwindigkeit ermittelt und visualisiert werden.

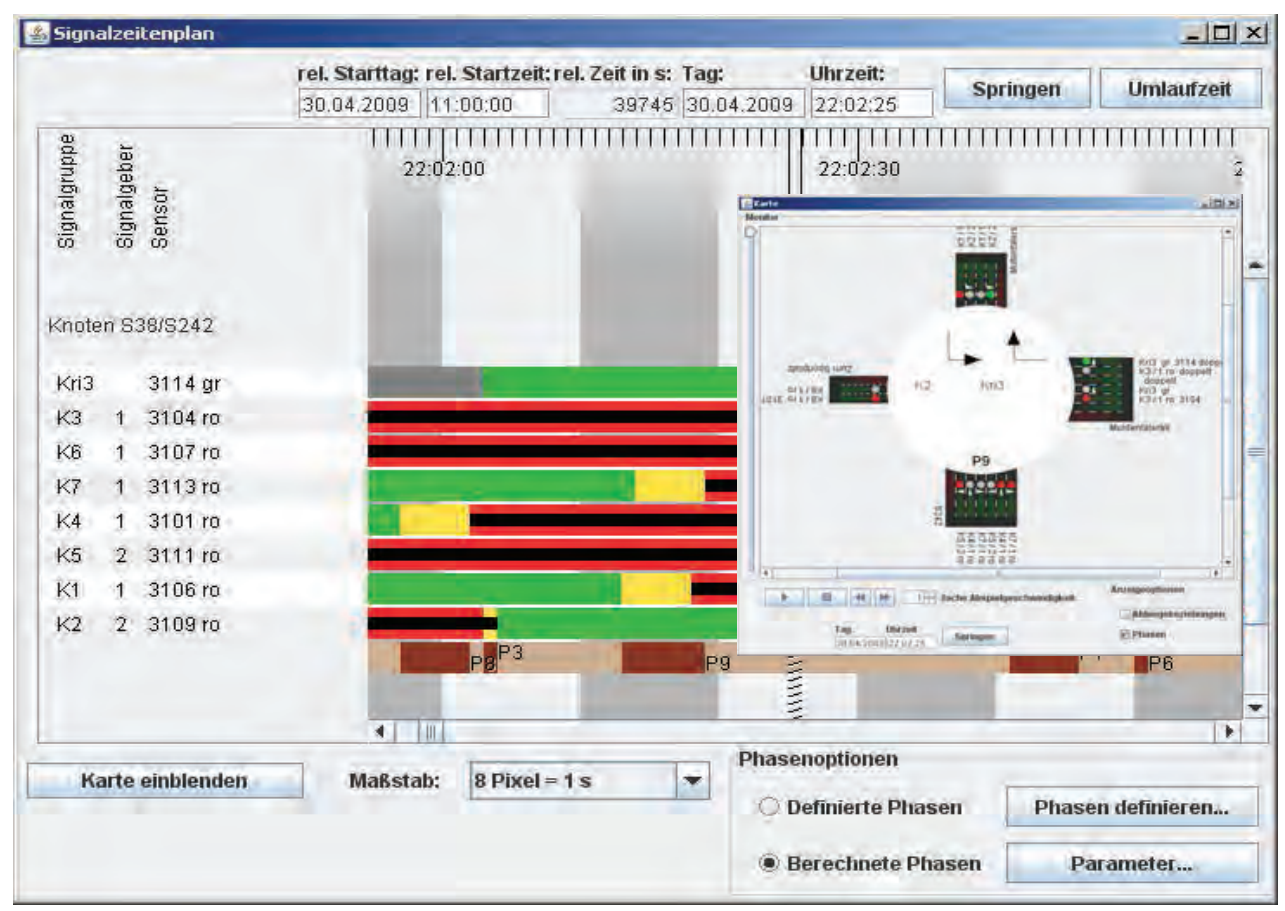

Abbildung 8: Aufbereitete Sensordaten einer Messkampagne

Das Monitoring-System dient vorrangig der Qualitätsanalyse von Lichtsignalanlagen. Aus der Beobachtung der Signalgeber-Schaltzustände wird auf die Funktionsweise der gesamten Anlage geschlossen. So werden Abweichungen vom Planungsstand herstellerunabhängig, rückwirkungsfrei und ohne Eingriff in die Anlage aufgedeckt.

Das Monitoring-System eignet sich aber auch dazu, eine vereinfachte Ersatzsteuerung beispielsweise für Verkehrssimulationsmodelle zu generieren, in denen die oftmals sehr komplexen verkehrsabhängigen Steuerungsverfahren aus Zeit- und Aufwandsgründen nicht adäquat nachgebildet werden können.

\section{Kommunikation zwischen Fahrzeugen und Infrastruktur im urbanen Raum}

\section{Anforderungen an die Fahrzeug-Infrastruktur-Kommunikation}

Das Fahrzeug wandelt sich zunehmend vom Empfänger zum Sensor und Sender von Verkehrsinformationen. Die technologische Grundlage bilden neben Sensor- und Aktorsystemen im Fahrzeug die modernen Kommunikationseinrichtungen, mit denen der Daten- und Informationsaustausch zwischen Fahrzeugen und Infrastruktur (Car2X-Kommunikation) erst möglich wird. Durch diese kooperative Anwendung soll ein Lückenschluss zwischen den Erfassungsbereichen aktueller On-BoardSysteme und den infrastrukturseitig vorliegenden Informationen über die Verkehrslage erreicht werden. Fahrzeuge sollen zukünftig spontan Kommunikationsnetzwerke aufbauen und ihre relevanten 
Informationen für sicherheitsrelevante Applikationen austauschen und weitergeben können. Die Automobil- und Zulieferindustrie hat somit ein gesteigertes Interesse an der Nutzung qualitativ hochwertiger, externer Daten. In sich spontan ergebenen Vehicular Ad Hoc Networks (VANETs) kann eine Vielzahl von Verkehrsteilnehmern bei hohen Geschwindigkeiten und damit kurzen Kommunikationsfenstern dynamische Informationen austauschen. Es sollen sowohl sicherheits- als auch serviceorientierte Applikationen ermöglicht werden, die im direkten Austausch, oder über entsprechende Infrastruktur eine Vielzahl kooperativer Dienste bieten.

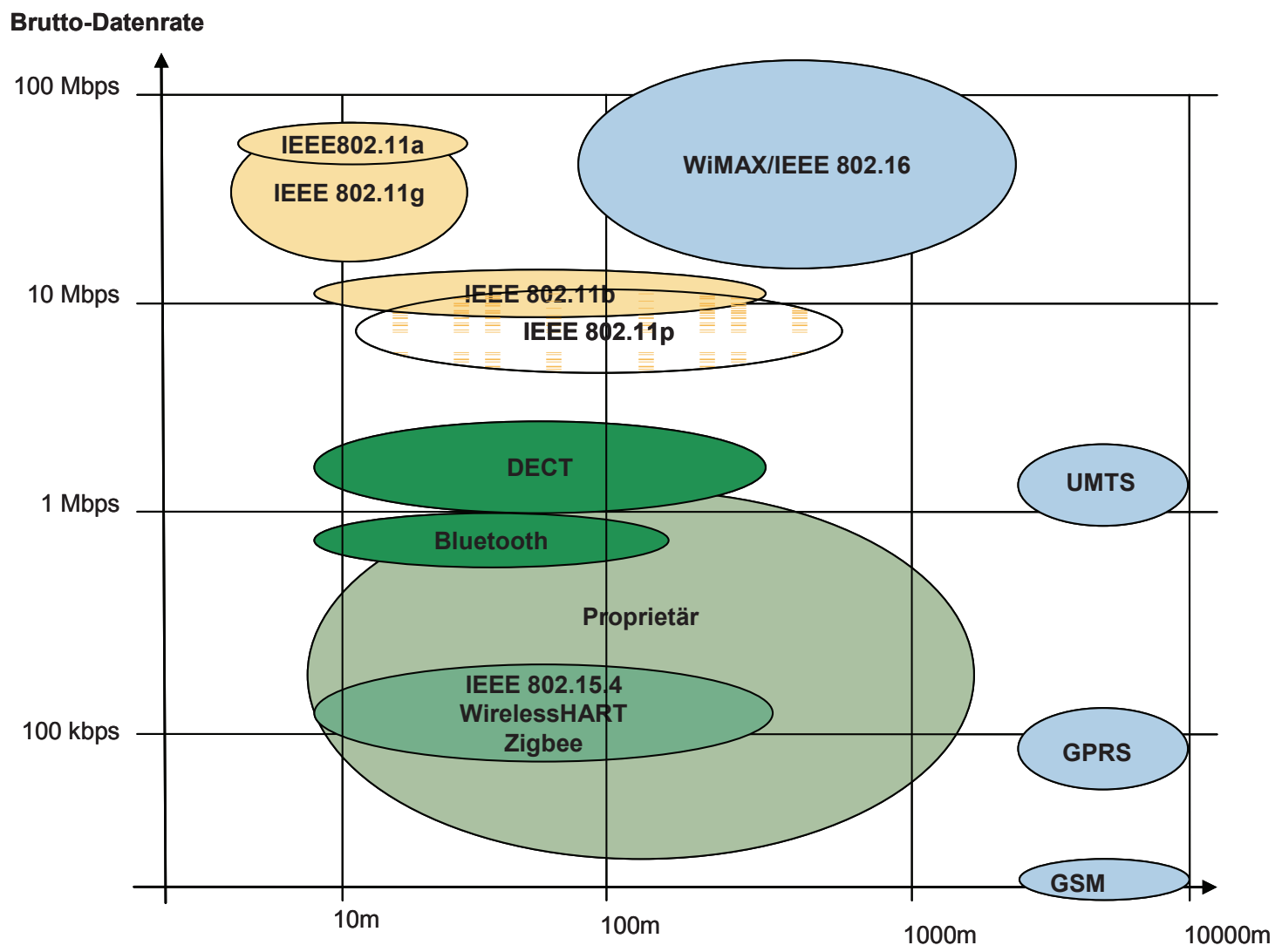

Abbildung 9: Übersicht über aktuelle Funkstandards im Verkehrsbereich

Für diese dezentrale Kommunikation zwischen Fahrzeugen und Infrastruktur können generell verschiedene Technologien mit unterschiedlichen Reichweiten und Datenraten genutzt werden (Abbildung 9). Für den Datentransfer zwischen Verkehrssensoren und Verkehrszentralen werden überwiegend noch mobilfunkgestützte Kommunikationssysteme zur Datenübertragung genutzt, für die Nahbereichskommunikation im direkten Fahrzeugumfeld gewinnen jedoch WLAN-basierte Funklösungen zunehmend an Bedeutung.

Aktuell bestehen eine Reihe von unterschiedlichen Ausprägungen des Wireless LAN-Standards IEEE 802.11. Der allgemein in Heimnetzwerken genutzte und vor allem in Europa etablierte WLAN-Standard ist der IEEE $802.11 \mathrm{~b} / \mathrm{g} / \mathrm{n}$, welcher das Frequenzband zwischen 2,400 und 2,485 GHz sowie $5 \mathrm{GHz}$ nutzt. Auf Basis dieses Standards sind aktuell vielseitige Anwendungen für zunehmend kostengünstige und leistungsstarke Endgeräte verfügbar. Allerdings ist eine direkte Übernahme von Funksystemstandards, wie sie aus dem Heim- und Office-Bereich bekannt sind, nicht ganz unproblematisch, da Car2XAnwendungen völlig neue Anforderungen an die Kommunikationssysteme stellen. Um den Kommunikationshorizont der Fahrzeuge mittels Funktechnologien ausreichend zu erweitern, wird vor allem außerorts und auf Autobahnen von notwendigen Funkreichweiten von bis zu $1000 \mathrm{~m}$ in und entgegen der Fahrtrichtung ausgegangen. Bei nominellen Reichweiten von WLAN IEEE 802.11g von bis zu 100 m, unter Nutzung spezifischer Antennen und Repeater bis zu 250m, zeigt sich hier noch eine deutliche Diskrepanz zwischen Anspruch und Realität. Bei derzeitigen Funkreichweiten befinden sich zwei Fahrzeuge, welche beide mit $100 \mathrm{~km} / \mathrm{h}$ aufeinander zu fahren, für max. $18 \mathrm{~s}$ in gegenseitiger Funkreichweite. Dieser relativ kurze Zeitraum stellt für den Austausch von Daten nach einem erforderlichen sicheren (funktional und informationstechnisch) Auf- und Abbau der Kommunikation selbst unter idealen Randbedingungen eine große Herausforderung dar. 
Wie wichtig die WLAN-Technologie für kooperative Systeme im Verkehr ist, zeigt sich in der Verabschiedung eines neuen WLAN-Standards, welcher die bestehenden Vorteile der aktuell vielseitig genutzten Technologie mit der Erweiterung spezieller Eigenschaften, wie Sicherheits- und Verfügbarkeitsanforderungen für kooperative Verkehrsanwendungen verbinden soll. Für die exklusive Nutzung der WLAN-Technologie im Verkehrsbereich wurde auch in Hinblick auf entsprechende internationale Standardisierungsbestrebungen ein neues Frequenzband definiert, welches die Anforderungen aller maßgeblichen internationalen Akteure bezüglich sicherheits- und zeitkritischer Anwendungen erfüllbar macht.

\section{Automotive-Funkstandard für kooperative Verkehrssysteme}

In Erweiterung der IEEE 802.11-Norm wurde Anfang 2009 der IEEE 802.11p-Draft für spezifische Applikationen der intelligenten Verkehrssysteme im Frequenzband von 5,85 bis 5,925 GHz vorgestellt. Im Rahmen von Harmonisierungsbestrebungen wurde ausgehend von der Frequenznutzung in Japan und den vereinigten Staaten seitens der Electronic Communications Committee (ECC) die Nutzung des 5,875-5,925 MHz Bandes vereinbart und der Europäischen Union vorgeschlagen. Dabei wird $30 \mathrm{MHz}$ im Bereich zwischen 5,875 und 5,905 GHz für sicherheitsrelevante Anwendungen der Car2X Kommunikation bereitgestellt (Abbildung 10). Durch Multikanalnutzung soll für die spezifischen Anforderungen künftiger Car2X-Kommunikation eine technologische Basis für die VANETs geschaffen werden. Vor allem für den Nahbereich bis 1000 m sollen so zukünftig Positionsdaten, Status- und Gefahrenmeldungen aber auch andere nützliche Informationen direkt zwischen Fahrzeugen und Infrastruktur ausgetauscht werden.

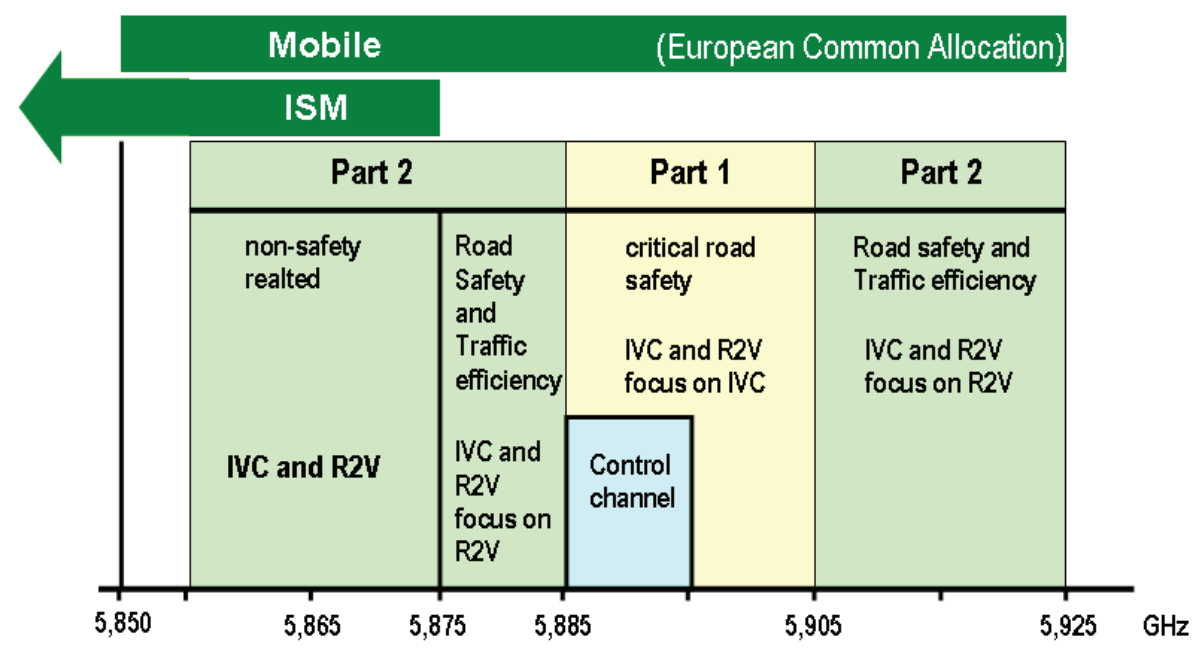

\section{Abbildung 10: Frequenznutzung des IEEE 802.11p in Europa}

Ein international nutzbarer, zukunftsfähiger, verlässlicher Kommunikationsstandard ist für die Marktdurchsetzung künftiger Applikationen die Automobilindustrie maßgeblich. Eine zukunftsweisende intelligente Verkehrstelematik kann nur dann erfolgreich sein, wenn auf lange Sicht hin ein spezifisches Spektrum exklusiv für die Fahrzeug-Fahrzeug und Fahrzeug-Infrastruktur-Kommunikation zur Verfügung gestellt und die entsprechende Funkkommunikation standardisiert wird.

\section{Anwendungsbeispiel Kooperative Lichtsignalanlage}

Die Höhe der Lärm- und Schadstoffemissionen des Kraftfahrzeugverkehrs wird wesentlich durch dessen Beschleunigungsvorgänge beeinflusst. Ein Großteil der Verzögerungs- und Beschleunigungsvorgänge wird im Stadtverkehr durch die Halte an Lichtsignalanlagen (LSA) verursacht. Gelingt es, durch eine gute Koordinierung der Steuerung von LSA und durch eine geeignete Kommunikation zwischen Fahrzeugen und Infrastruktur die Anzahl der Halte im Straßennetz zu minimieren, so ist eine Verbesserung bezüglich der Wartezeiten des Kfz-Verkehrs, der Emissionen insgesamt, der Immissionen im Nahbereich, der Kapazität an Knotenpunkten und der Anfahrverluste angehaltender Fahrzeugen zu erwarten.

Das ist ein wesentliches Ziel des Forschungsprojekts KOLINE (Kooperative und optimierte Lichtsignalsteuerung in städtischen Netzen). Das im Projekt entwickelte System umfasst alle notwendigen Komponenten und Teilsysteme, um eine Kooperation von Fahrzeugen und Infrastruktur über ein geeignetes Kommunikationsnetzwerk auf Basis des IEEE 802.11p-WLAN-Standards zu ermöglichen. 
Durch den bidirektionalen Datenaustausch zwischen Fahrzeug und LSA stehen beiden Seiten bisher nicht verfügbare Informationen hoher Güte zur Verfügung. Diese werden fahrzeugseitig durch

Assistenzsysteme zur Optimierung der Annäherung an den signalisierten Knotenpunkt und auf Seiten der Infrastruktur für eine verbesserte Verkehrszustandsanalyse sowie die Bestimmung einer dem aktuellen Verkehrszustand angepassten LSA-Steuerung genutzt.

Der Fokus der weiteren Arbeiten der Forschungspartner liegt in der Implementierung und Erprobung des Gesamtsystems. Die Anwendung und Evaluierung des Systems auf der Straße ist ein entscheidender Schritt, um den Nachweis der Funktionsfähigkeit und der Effizienz eines kooperativen Verkehrssystems im Hinblick auf ausgestattete bzw. auch nicht ausgestattete Fahrzeug sowie das gesamte verkehrliche Umfeld zu erbringen (s. a. [9]).

\section{Kooperative und intelligente Kreuzungen - Ein kurzer Ausblick}

Die Grundprinzipien des heutigen Verkehrsmanagements basieren auf der Messung und Steuerung der verschiedenen Verkehrsströme. Der Verkehrsfluss in urbanen Netzen und damit die Verkehrsqualität und -effizienz werden maßgeblich von Lichtsignalsteuerungen (LSA) beeinflusst. Mit geeigneten dynamischen LSA-Steuerungsstrategien ist es möglich, die stärksten Verkehrsströme auf dem Hauptstraßennetz zu bündeln, besonders umweltfreundliche Verkehrsarten, wie den öffentlichen Personennahverkehr (ÖPNV), gezielt zu bevorrechtigen, Halte- und Reisezeiten zu reduzieren und so auch Lärm- und Schadstoffemissionen zu minimieren. Voraussetzung hierfür ist die Verfügbarkeit von Verkehrsdaten in hoher örtlicher und zeitlicher Auflösung.

Diese werden zunehmend durch komplexe Sensor- und Kommunikationsnetzwerke gewonnen und weitergeleitet. Zukünftig sollen auch verstärkt Fahrzeuge Teil dieser Netzwerke sein. Gegenwärtig können Fahrzeuge bereits mittels umfassender On-Board-Sensoren wie Radar- und Wettersensoren, Heckkameras und Straßensensoren ihre direkte Umgebungssituation erfassen. Nutzen Fahrer- und Fahrzeugassistenzsysteme für die Datengewinnung bisher vor allem diese bordeigenen Sensoren, greifen Fahrerinformationssysteme vor allem auf von Verkehrszentralen und Meldestellen zur Verfügung gestellte Daten zu. Umgekehrt könnte zukünftig auch die Infrastruktur diese hochgenauen fahrzeugseitigen Verkehrsdaten nutzen um noch besser auf bestehende Situationen reagieren zu können. Eine entscheidende Grundvoraussetzung für diese Kooperation ist neben der zuverlässigen und möglichst vollständigen Datenerfassung ein effizientes und sicheres Kommunikationsnetzwerk zwischen Fahrzeugen untereinander und der straßenseitigen Verkehrsinfrastruktur.

Aktuelle Forschungsprojekte wie simTD (Sichere Intelligente Mobilität - Testfeld Deutschland, www.simtd.org) untersuchen die Möglichkeiten und Grenzen der Fahrzeug-Infrastruktur-Kommunikation mit realitätsnahen Verkehrsszenarien in einer großflächigen Testfeld-Infrastruktur rund um die hessische Metropole Frankfurt/Main. Dabei werden die politischen, wirtschaftlichen und technologischen Rahmenbedingungen für eine erfolgreiche Einführung der Fahrzeug-zu-Fahrzeug- und Fahrzeug-zuInfrastruktur-Vernetzung untersucht.

Im Anfang 2012 gestarteten Forschungsvorhaben UR:BAN (Urbaner Raum: Benutzergerechte Assistenzsysteme und Netzmanagement) werden innovative Fahrerassistenz- und Verkehrsmanagementsysteme für urbane Räume entwickelt und getestet und deren Beitrag zur Verbesserung der Sicherheit und Effizienz bewertet. Durch Ausbau von intelligenter Infrastruktur und deren Vernetzung mit intelligenten Fahrzeugen soll die Verkehrseffizienz in urbanen Räumen bei gleichzeitiger Senkung des Emissionsausstoßes optimiert werden. Dabei werden auch verschiedene Antriebskonzepte wie Elektro- und Hybridantriebe berücksichtigt. Ein weiterer Aspekt ist die Betrachtung des Menschen mit seinen vielfältigen Rollen im Verkehrssystem.

Wenn es zukünftig mit Hilfe kooperativer Systeme, Anwendungen und Sensoren gelingt, die vielfältigen Nutzungsansprüche der verschiedenen Verkehrsteilnehmer in Städten besser zu vereinbaren, die durch die begrenzte Verkehrsinfrastruktur entstehenden Konflikte zu begrenzen und die Informationsbasis durch verteilte, standardisierte Sensor- und Kommunikationsnetzwerke signifikant zu verbessern, können Intelligente Verkehrssysteme einen sichtbaren Beitrag zum Umwelt- und Klimaschutz sowie zur Reduktion von Schadstoffemissionen und Lärm in Städten und Ballungsräumen leisten.

\section{Danksagung}

Die Autoren danken dem Bundesministerium für Wirtschaft und Technologie (BMWi) und dem Bundesministerium für Bildung und Forschung (BMBF) für die Förderung der Forschungs- und Entwicklungsarbeiten in den Projekten VAGABUND, AKTIV, MOSAIQUE, SAFE, MD-E4 und KOLINE (Förderung aufgrund eines Beschlusses des Bundestages unter den Förderkennzeichen 19B5019A, 19P6018N, 19B6008A, 01LS05062, 03SF0407E und 19P9002D). 


\section{Literatur:}

[1] Mitteilung der Europäischen Kommission, „Aktionsplan für die Einführung intelligenter Verkehrssysteme in Europa", $\operatorname{COM(2008)~886,~Brüssel,~16.12.2008.~}$

[2] Richtlinie 2010/40/EU des Europäischen Parlaments und des Rates vom 7. Juli 2010 zum Rahmen für die Einführung intelligenter Verkehrssysteme im Straßenverkehr und für deren Schnittstellen zu anderen Verkehrsträgern, Brüssel, 07.07.2010.

[3] Jenkner, S.; Herrmann, A.: "The ITS Master Plan of the German Federal State of Saxony-Anhalt", Technical Paper, ITS Europe Congress, Lyon, Proceedings, 2011.

[4] Naumann, S.; Schönrock, R.: „Floating Car Observer - Ein preisgünstiger Sensor zur Gegenverkehrsbeobachtung“. 11. Braunschweiger Symposium AAET - Automatisierungssysteme, Assistenzsysteme und eingebettete Systeme für Transportmittel, Braunschweig, 10.-11.02.2010. ISBN 978-3-937655-23-9.

[5] Schulz, E.; Naumann, S.; Schönrock, R.: Stauerkennung und Staulängenmessung mittels Floating Car Observer. Automation 2010, Baden-Baden, 15.-16.06.2010. VDI-Berichte Nr. 2092 (2010), ISBN 978-3-18-092092-4, S. 357-359

[6] Wolf, F.; Libbe, S.; Herrmann, A.: Co-operative traffic light - Applications for Driver Information and Assistance. 3rd International Workshop on Intelligent Vehicle Controls \& Intelligent Transportation Systems on 6th International Conference on Informatics in Control, Automation and Robotics, Milano/Italy, 4.-5.07.2009. Proceedings on CD.

[7] Schade, J.; Schimschar, A.; Rauchhaupt, L.: Leistungsbewertung eines Funkkommunikationssystems nach IEEE 802.11p unter dem Aspekt verkehrsbezogener Anwendungen (C2X) - Performancetests von Car-to-Car-Anwendungen. Automation 2011, Baden-Baden, 26.06.2011. Proceedings

[8] Lehnhoff, N.: „Überprüfung und Verbesserung der Qualität von automatisch erhobenen Daten an Lichtsignalanlagen“, Heft 34, Institut für Verkehrswirtschaft, Straßenwesen und Städtebau der Universität Hannover, Juli 2005.

[9] Bley, O.; Kutzner, R.; Friedrich, B.; Saust, F.; Wille, J.; Maurer, M.; Niebel, W.; Naumann, S.; Wolf, F.; Schüler, T.; Bogenberger, K.; Junge, M.; Langenberg, J.: Kooperative Optimierung von Lichtsignalsteuerung und Fahrzeugführung, 12. Braunschweiger Symposium AAET Automatisierungssysteme, Assistenzsysteme und eingebettete Systeme für Transportmittel, 9.10.2.2011 